\title{
Effects of dietary supplementation with essential oils and organic acids on the growth performance, immune system, fecal volatile fatty acids, and microflora community in weaned piglets
}

\author{
Caimei Yang, ${ }^{*, 1}$ Lingling Zhang, ${ }^{*, 1}$ Guangtian Cao, ${ }^{\dagger, 2}$ Jie Feng, ${ }^{\ddagger}$ Min Yue, ${ }^{\ddagger}$ Yinglei Xu, ${ }^{*}$ \\ Bin Dai,* Qianjie Han,* and Xiqin Guo* \\ ${ }^{*}$ College of Animal Science and Technology, Zhejiang A \& F University, Hangzhou 311300, China; ${ }^{\dagger C}$ College of \\ Standardisation, China Jiliang University, Hangzhou 310018, China; and ${ }^{\ddagger}$ College of Animal Science, Zhejiang \\ University, Hangzhou 310058, China
}

\begin{abstract}
The present study was conducted to assess the effects of a mixture of essential oils and organic acids on the growth performance, immune system, major fecal volatile fatty acids (VFAs), and microflora community in the weaned piglets. We also evaluated the antibacterial activity of the essential oil mixture on Escherichia coli and Staphylococcus aureus. Three hundred weaned piglets (Duroc $\times$ Landrace $\times$ Yorkshire) were randomly divided into the following 3 treatment groups: basal diet $(\mathrm{C})$, basal diet supplemented with the mixture of essential oils and organic acids (T1), and basal diet supplemented with antibiotics (T2). The mixture of essential oils and organic acids comprised of cinnamaldehyde $(15 \%)$, thymol $(5 \%)$, citric acid $(10 \%)$, sorbic acid $(10 \%)$, malic acid (6.5\%), and fumaric acid (13.5\%). In vitro studies showed that the mixture of essential oils extremely damaged the cell structure of pathogenic bacteria by deforming the membranes and disorganizing the intracellular components. In vivo studies revealed that diet supplementation with a mixture of essential oils and organic acids improved the final body weight and ADG of piglets $(P<0.05)$, increased the concentration of
\end{abstract}

serum complement $4(P<0.05)$, and enhanced the fecal level of isovaleric acid $(P<0.05)$ compared with controls on day 28. Result of high-throughput sequencing revealed that: 1) a total of 1,177 and 1,162 observed taxonomic units (OTUs) were shared between all treatment groups on day 14 and 28, respectively; 2) the T1 exhibited higher $(P<0.05)$ beta diversity (unweighted UniFrac distance) than control and antibiotics treatment on day $28 ; 3)$ the samples in principle component analysis plot and tree of relative abundance were separated from each other based on dietary treatments and age; 4) Firmicutes and Bacteroidetes were the most 2 dominate phyla; Lactobacillus and Streptococcus were the 2 top species among the recognized microbiota; 5) T1 had higher $(P<0.05)$ relative abundance of Lactobacillus mucosae than control and antibiotics treatment on day 28 . To conclude, the mixture of cinnamaldehyde and citric acids damaged the structure of pathogens in vitro; the mixture of essential oils and organic acids improved the growth performance, increased the fecal concentration of isovaleric acid, and modulated the microflora community in weaned piglets.

Key words: essential oils, fecal microflora, growth performance, immune system, organic acids, weaned piglets

(C) The Author (s) 2018. Published by Oxford University Press on behalf of the American Society of Animal Science. All rights reserved. For permissions, please e-mail: journals.permissions@oup.com.

J. Anim. Sci. 2019.97:133-143 doi: 10.1093/jas/sky426

\footnotetext{
${ }^{1}$ These authors contributed equally to this work. ${ }^{2}$ Corresponding author: 15a1903025@cjlu.edu.cn Received September 9, 2018.

Accepted October 31, 2018.
}

\section{INTRODUCTION}

Weaning often brings a series of problems during the growth of piglets, such as high incidence of diarrhea, decreased feed intake, and 
growth performance (Boudry et al., 2004). Several studies on potential alternatives for antibiotics are being conducted on livestock. Essential oils, which are natural bioactive compounds derived from plants, have been shown to effectively replace the use of antibiotics, and play key roles in the growth and development of livestock (Pathak et al., 2016; Valenzuela-Grijalva et al., 2017). In swine production, diets supplemented with different kinds of the essential oils are effective in improving growth performance, antioxidant potential, and immune system, and modulating intestinal health and decreasing the incidence of diarrhea. Boudry and Perrier (2008) observed that thymol and cinnamaldehyde may exert an antioxidant effect in the small intestine of weaned piglets. Moreover, several studies have concluded that organic acids could improve the growth performance and reduce diarrhea in weaned pigs by lowering the digesta $\mathrm{pH}$ and maintaining the balance of microflora (Torrallardona et al., 2007; Diao et al., 2014; Suiryanrayna and Ramana 2016). In addition, in view of minimum inhibitory concentration, cost-effectiveness and feed palatability, essential oils are effective bacteriostatic in animal industry (Yang et al., 2015). Moreover, in vitro trials have confirmed the antimicrobial activity of a mixture essential oils containing thymol, eugenol, and carvacrol have high antimicrobial activity against pathogenic bacteria such as Escherichia coli, Staphylococcus aureus, and Salmonella typhimurium (Mulyaningsih et al., 2010; Hippenstiel et al., 2011; Bassole and Juliani, 2012). Although many beneficial effects of essential oil or organic acids diet supplementation have been reported, few studies assessed the synergistic antibacterial effects in weanling piglets (Amorati et al., 2013; Cairo et al., 2018). Hence, the aim of this study was to assess the synergistic effects of a mixture of essential oils and organic acids, which could treated as potential alternatives for antibiotics, on the growth performance, immune system and fecal microbial community of weaned piglets. We also aim to investigate the in vitro antibacterial effects of essential oils mixture.

\section{MATERIALS AND METHODS}

\section{Animals and Treatments}

The experimental protocol was approved by the Ethics Committee of Zhejiang A \& F University (Hangzhou, China). At weaning $(21 \pm 1 \mathrm{~d})$, a total of 300 crossbred piglets (Duroc $\times$ Landrace $\times$ Yorkshire) were randomly allotted into 3 treatment groups with 10 pens per treatment, and 10 animals per pen. Piglets were housed in 3 separate rooms with 10 -floor pens. The study lasted for $28 \mathrm{~d}$. The control group received basal diet $(\mathrm{C})$; T1 group received basal diet with combination of plant essential oils and organic acids $(1 \mathrm{~kg} / \mathrm{t})$; T2 group received basal diet with terramycin $(1 \mathrm{~kg} / \mathrm{t})$. The diet was formulated to be isonutritive, exceeding the protein requirement recommended by NRC (1998) for pigs (Table 1), and did not contain antibiotic growth promoters. Water and feed were provided ad libitum. The starting temperature inside the pens was set at $28.0^{\circ} \mathrm{C}$; the temperature reduced gradually at a rate of $0.5^{\circ} \mathrm{C}$ per week and reached a final temperature of $26^{\circ} \mathrm{C}$ at the end of the study.

\section{Preparation of Essential Oils and Organic Acids Supplemented Feed}

The mixture of essential oils and organic acids comprised of cinnamaldehyde $(15 \%)$, thymol $(5 \%)$, citric acid (10\%), sorbic acid (10\%), malic acid (6.5\%), and fumaric acid (13.5\%). The mixture was enveloped with stearic acid, and packaged as microcapsules by the sprayed-drying technology (Vegamax Biotechnology Co. Ltd., Anji, China). The air inlet temperature was $12^{\circ} \mathrm{C}$ and the air outlet temperature was $26^{\circ} \mathrm{C}$.

\section{Strains}

E. coli (ATCC25922) and $S$. aureus (ATCC25923) are purchased from China Centre of Industrial Culture Collection (CICC). Bacterial suspensions were prepared daily by their suspending colonies in sterile PBS solution.

Table 1. Compositions and nutrient levels of the basal diet (air-dry basis) \%

\begin{tabular}{lrcr}
\hline \hline Ingredients & Content & Nutrient level & \\
\hline Corn & 54.70 & Gross Energy, MJ/kg & 13.99 \\
Wheat middling & 3.00 & Crude Protein & 19.55 \\
Soybean meal & 18.20 & Lys & 1.30 \\
Extruded soybean & 8.00 & Met & 0.39 \\
Fish meal & 5.00 & Met+Cys & 0.70 \\
Whey & 5.00 & Thr & 0.77 \\
Choline chloride & 0.10 & Calcium & 0.94 \\
Phospholipid & 2.00 & Total Phosphorus & 0.70 \\
Premix $^{1}$ & 4.00 & Available Phosphorus & 0.46 \\
Total & 100 & & \\
\hline
\end{tabular}

${ }^{1}$ Supplied the following per $\mathrm{kg}$ of diet: vitamin A, 10,000 IU; vitamin D3, $400 \mathrm{IU}$; vitamin E, $10 \mathrm{mg}$; pantothenic acid, $15 \mathrm{mg}$; vitamin B6, $2 \mathrm{mg}$; biotin, $0.3 \mathrm{mg}$; folic acid, $3 \mathrm{mg}$; vitamin B12, $0.009 \mathrm{mg}$; ascorbic acid, $40 \mathrm{mg}$; Fe, $150 \mathrm{mg}$; Cu, $130 \mathrm{mg}$; $\mathrm{Mn}, 60 \mathrm{mg}$; Zn, $120 \mathrm{mg}$; I, $0.3 \mathrm{mg}$; Se, $0.25 \mathrm{mg}$.

${ }^{2}$ Nutrient levels are calculated. 


\section{Growth Performance}

All pigs were weighed individually at the beginning and end of the experiment. Residual feed were weighed every day. Average daily gain, ADFI, and feed:gain $(F: G)$ were calculated for each pen. Diarrhea rate was calculated according to the formula reported by Sun et al. (2008): Diarrhea rate $(\%)=$ the number of diarrhea pigs $\times$ diarrhea days/the total number of pigs $\times$ experiment days. The diarrhea rate scoring consulted the previous study (Long et al., 2018), and diarrhea signals were manually observed every day (score 0, hard feces; score 1, slightly soft feces; score 2, soft, partially formed feces; score 3, loose, semiliquid feces; score 4, watery, mucous-like feces).

\section{Samples Collection}

On days 14 and 28, 1 pig per pen was randomly selected for the collection of blood. Blood samples were obtained into the heparinized tubes from the front cavity vein; the serum was separated and immediately stored at $-20{ }^{\circ} \mathrm{C}$ for further analysis. Early in the morning, fresh feces were collected into sterile sample bags from each pen and stored at $-80^{\circ} \mathrm{C}$ for the detection of VFAs and for high-throughput sequencing.

\section{Morphological Analysis of Bacteria}

The bactericidal action of cinnamaldehyde and citric acid was performed by the method of Liu et al. (2009), with slight modifications. E. coli and S. aureus were cultured in sterile lysogeny broth (LB) for $10 \mathrm{~h}$ and then treated with cinnamaldehyde $(1,000$ $\mathrm{IU} / \mathrm{mL})$, citric acid $(1,000 \mathrm{IU} / \mathrm{mL})$, and a mixture of cinnamaldehyde and citric acid $(1,000 \mathrm{IU} / \mathrm{mL})$ at $37^{\circ} \mathrm{C}$ for $8 \mathrm{~h}$. The cells were then washed twice with the sterile PBS to remove the cinnamaldehyde and citric acid. After centrifugation, pellets were cut into 85-nm-thick sections using a Leica A-1170 (Leica, Germany). The sections were placed onto 200-mesh thin bar grids and poststained for $20 \mathrm{~min}$ with 5\% uranyl acetate and 10 min with Sato's triple lead stain. The stained samples were observed under a transmission electron microscope (Model H-7650, Hitachi, Japan) operating at $80 \mathrm{kV}$.

\section{VFA Analysis}

VFAs were analyzed by Headspace Sampler Gas Chromatography (Agilent Technologies, New Castle, DE) using the method of Thanh et al. (2009), with slight modifications. The commercial standards of acetic acid, propionic acid, isobutyric acid, butyric acid, isovaleric acid, and valerate, used as external standards, were purchased from Aladdin Biochemical Polytron Technologies Inc. (Shanghai, China). Metaphosphoric acids were obtained from China National drug group chemical reagents Co. Ltd., (Beijing, China). One-gram fecal content was blended with $6 \%$ phosphorous acid $(\mathrm{m} / \mathrm{v}, 1: 4)$, and was injected into an Agilent Technologies $6890 \mathrm{~N}$ Network System (Agilent Technologies) equipped with a $30 \mathrm{~m} \times 0.25 \mathrm{~mm} \times 0.25 \mu \mathrm{m}$ column $($ DB-FFAP, Agilent Technologies) and a flame ionization.

\section{Serum Parameter Analysis}

$\operatorname{Ig} \mathrm{A}, \operatorname{IgM}, \operatorname{IgG}$, and tumor necrosis factor- $\alpha$ $(\mathrm{TNF}-\alpha)$ were assayed using ELISA kits from Cusabio Biotech Co., Ltd. (Wuhan, China) following the manufacturer's instructions. Complements 3 and 4 (C3, C4) were detected using kits from Nanjing Jiancheng Bioengineering Institute, China following the manufacturer's instructions.

\section{Fecal Microflora 16S rRNA Sequencing}

Fecal samples from each pen were separately collected in the morning for microbial community analysis. The microbial genomic DNA was isolated using the PowerFecal Fecal DNA Kit (MoBio Laboratories, Inc., USA). The Illumina HiSeq platform, Novogene Bioinformatics Technology Co., Ltd. (Beijing, China), was used for the analysis of V4 region of 16S rRNA gene. The analysis was performed following the method of Zhu et al. (2018). Briefly, Quantitative Insights Into Microbial Ecology (QIIME) and the UPARSE software were used to assign taxonomy at $97 \%$ similarity with the RDP Classifier. The specific primer sequences were 515F (5'-GTGCCAGCMGCCGCGGTAA-3') and 806R ( $5^{\prime}$-GGACTACHVGGGTWTCTAAT-3'). Alpha and beta diversity measurement (unweighted and weighted UniFrac distance) were calculated using QIIME software. Principle component analysis (PCA) and non-metric multidimensional scaling analysis were performed to assess the clustering of fecal samples.

\section{Statistical Analysis}

Statistical analyses were performed using GraphPad Prism 6 software (GraphPad Software Inc., La Jolla, CA) and SPSS Statistics 16.0 Software Package (SPSS Inc., Chicago, IL). Oneway ANOVA was conducted to generate an estimate of the SEM. Significance was accepted at $P<0.05$. For comparative analysis of growth performance data, pen was considered as the experimental unit. For the other data, individual pig was treated as the experimental unit. 


\section{RESULTS}

\section{Antibacterial Properties of Cinnamaldehyde and Citric Acid}

We evaluated the effects of cinnamaldehyde and citric acid and a mixture of both on $S$. aureus and $E$. coli using transmission electron microscopy (Figs. 1 and 2). transmission electron microscopy revealed normal cell morphology and intact intracellular contents in $S$. aureus and $E$. coli maintained in the control growth media (Fig. 1A, a and Fig. 2A, a). On the contrary, E. coli cells treated with cinnamaldehyde and citric acid were damaged and showed an altered morphology. Similarly, S. aureus treated with cinnamaldehyde and citric acid showed extreme cellular damage with deformed membranes and damaged and disorganized intracellular structures (Fig. 1B,b, C, c and Fig. 2B, b, C, c). Furthermore, the destroyed cellular structures of E. coli and $S$. aureus were more seriously by the mixture of cinnamaldehyde and citric acid than that of the treatment of cinnaldehyde or citric acid alone (Figs. 1D, d and Fig. 2D, d).

\section{Effects of the Mixture of Essential Oils and Organic Acids on the Growth Performance of Weaned Piglets}

Effects of the mixture of essential oils and organic acids on the growth performance of weaned piglets are shown in Table 2. The T1 and T2 piglets had significantly higher ADG and final BW $(P<0.05)$ compared with control. No effects of diet supplementation with a mixture of essential oils and organic acids on ADFI and diarrheal incidence rate were observed in the piglets.

\section{Effects of the Mixture of Essential Oils and Organic Acids on the Fecal Concentration of VFAs in Weaned Piglets}

Effects of diet supplementation of the mixture of essential oils and organic acids on the fecal concentration of VFAs piglets are shown in Fig. 3. No significant changes in acetic acid, propionic acid, isobutyric acid, butyrate, and valeric acid concentrations were observed between the control and treatment groups on day 14 (Fig. 3A-F). On day 28, the concentration of isovaleric acid in T1 was significantly higher $(P<0.05)$ than the control and T2 piglets (Fig. 3K).

\section{Effects of the Mixture of Essential Oils and Organic Acids on the Serum Immunoglobulins, Complements, and TNF- $\alpha$ in Weaned Piglets}

The effects of diet supplementation on serum immunoglobulins, complements, and TNF- $\alpha$ are shown in Table 3. No significant effect on the overall concentrations of immunoglobulins throughout the study period was observed. Compared with the control piglets, T1 piglets showed a higher concentration of $\operatorname{IgG}$ and $\operatorname{IgM}(P>0.05)$ on day 14 , while no effects on IgA and IgG on day 28 were observed. Interestingly, the mixture of essential oils and organic acids significantly improved the concentration of $\mathrm{C} 4$ compared with the control piglets on
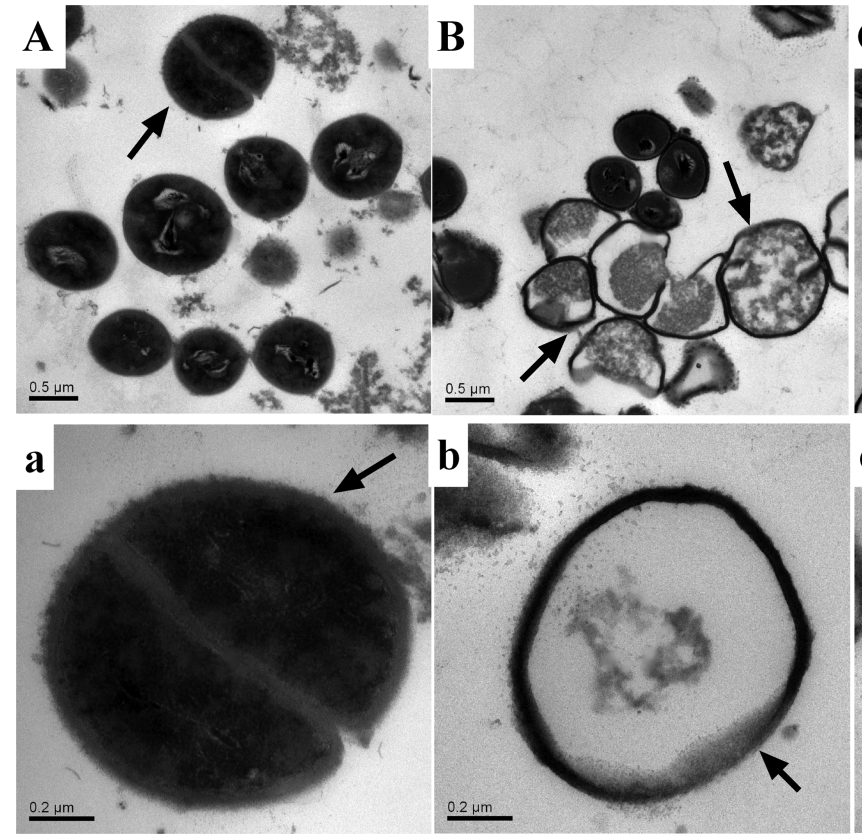
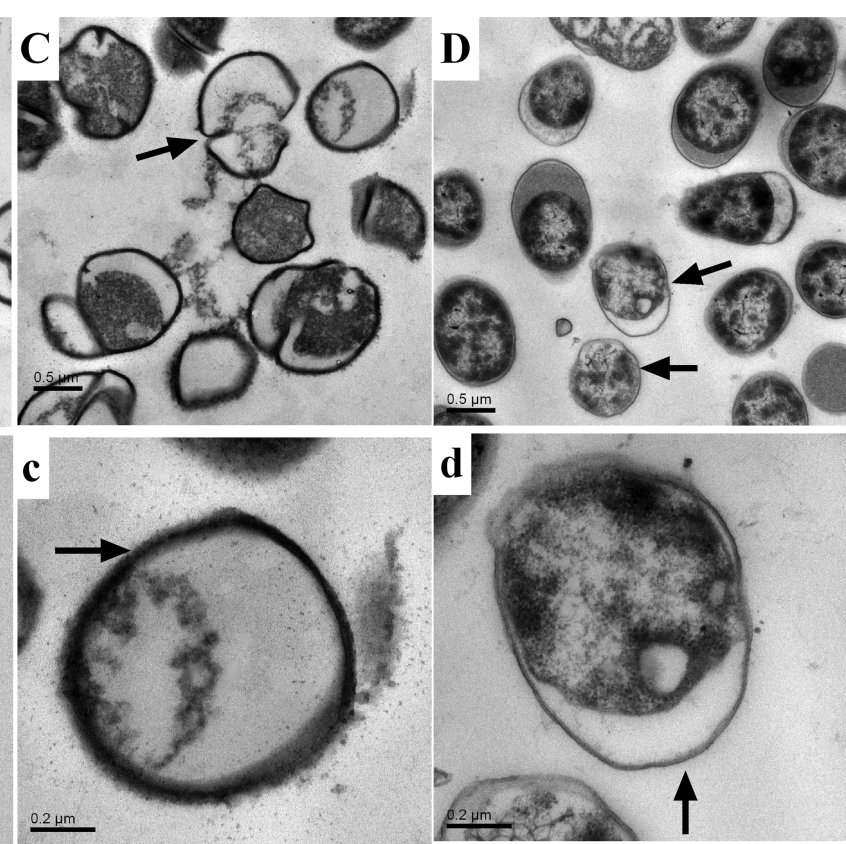

Figure 1. Transmission electron microscopy of $S$. aureus treated with cinnamaldehyde and citric acid. A, a represents $S$. aureus treated with LB after 8 h; B, b represents $S$. aureus treated with cinnamaldehyde after 8 h; C, c represents $S$. aureus treated with citric acid after 8 h; D, d represents $S$. aureus treated with cinnamaldehyde and citric acid after $8 \mathrm{~h}$. 

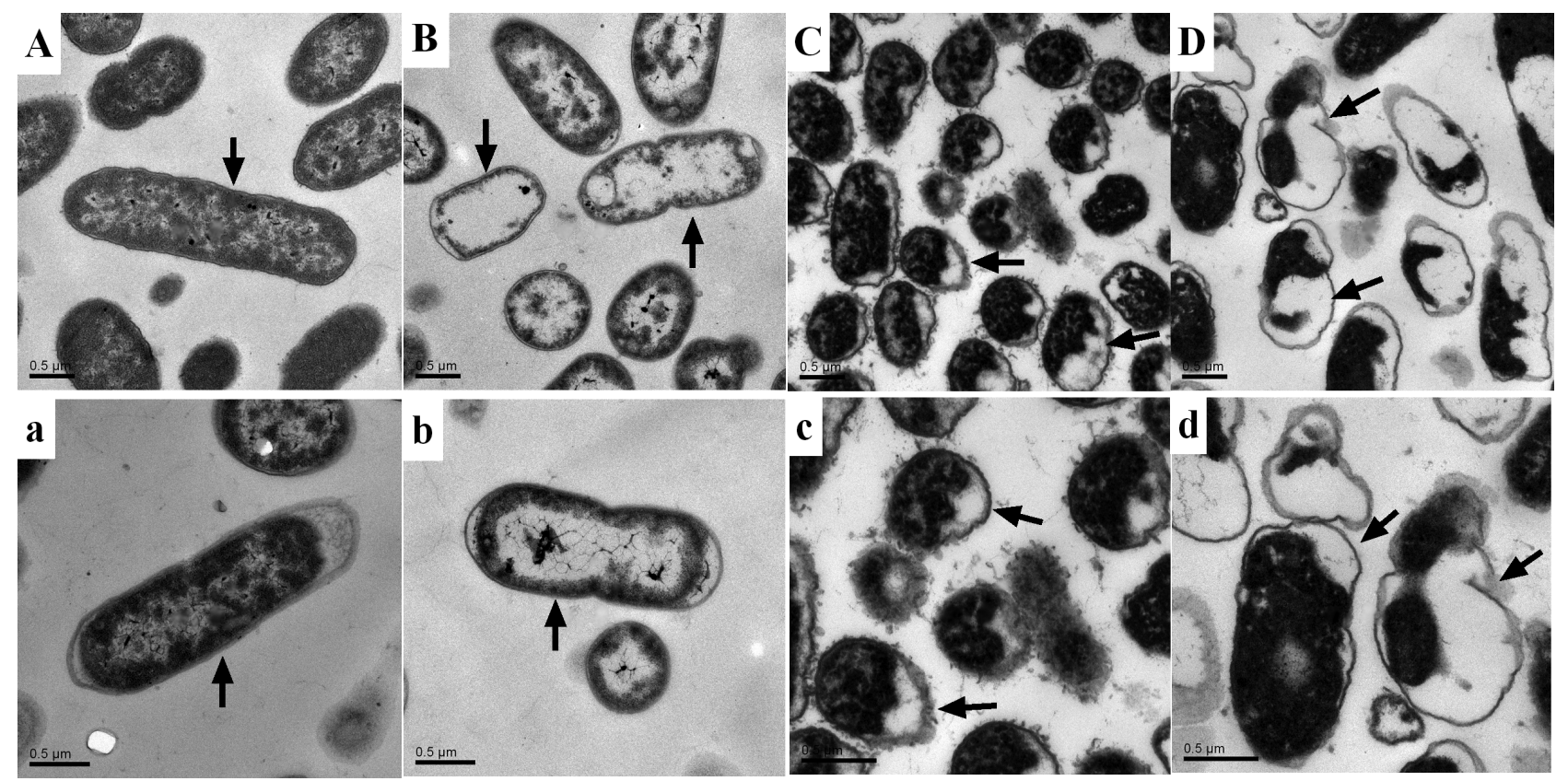

Figure 2. Transmission electron microscopy of $E$. coli treated with cinnamaldehyde and citric acid. A, a represents $E$. coli control treated with LB after 8 h; B, b represents $E$. coli treated with cinnamaldehyde after 8 h; C, c represents $E$. coli treated with citric acid after 8 h; D, d represents E. coli treated with the combination of cinnamaldehyde and citric acid.

Table 2. Effects of the combination of essential oils and organic acids on growth performance of the weaned piglets ${ }^{1}$

\begin{tabular}{lccccc}
\hline \hline & \multicolumn{3}{c}{ Treatment } & & \\
\cline { 2 - 5 } Item & $\mathbf{C}$ & T1 & T2 & SEM $^{2}$ & $P$ value \\
\hline Initial BW, kg & 6.13 & 6.10 & 6.11 & 0.11 & 0.99 \\
Final BW, kg & $10.66^{\mathrm{b}}$ & $11.26^{\mathrm{a}}$ & $11.41^{\mathrm{a}}$ & 0.14 & 0.04 \\
ADG, g & $162^{\mathrm{b}}$ & $184^{\mathrm{a}}$ & $190^{\mathrm{a}}$ & 4.86 & 0.03 \\
ADFI, g & 264 & 262 & 283 & 5.25 & 0.18 \\
F:G & 1.63 & 1.43 & 1.51 & 0.04 & 0.13 \\
Diarrhea rate, \% & 2.53 & 2.34 & 2.39 & 0.06 & 0.35 \\
\hline
\end{tabular}

a,b Means within the same raw with different superscripts differ significantly $(P<0.05)$.

${ }^{1} \mathrm{C}, \mathrm{T} 1$, and $\mathrm{T} 2$ represents the piglets supplemented with basal diet (control), piglets supplemented with the mixture of essential oils and organic acids and piglets supplemented with antibiotics, respectively.

${ }^{2}$ Pooled SEM; $n=10$ per treatment.

day 28. Serum level of C3 on day 28 was enhanced in T1 piglets with a tendency towards significance $(P=0.10)$.

\section{Effects of the Mixture of Essential Oils and Organic Acids on the Fecal Microbial Community in Weaned Piglets}

The microbiota in the feces is shown in the Venn diagram (Fig. 4A). A total of 1,177 observed taxonomic units (OTUs) were shared between the 3 treatment groups on day 14; the $\mathrm{C} 1$ piglets had 113 unique OTUs, T1 piglets had 82, and T2 piglets had 60 unique OTUs. On day 28 (Fig. 4B), 1,162 OTUs, were identified across the 3 treatment groups; the C2 piglets had 60 unique OTUs, T1 piglets 92, and the T2 piglets had 95 unique OTUs. No obvious differences were observed in the alpha diversity (Shannon) of fecal microbial community between the study groups on day 14 and 28 (Fig. 4D and E). The simpson parameter also did not show any differences (Supplementary Figure 3A, B). On the other hand, T1 had significantly $(P<0.05)$ higher beta diversity (unweighted UniFrac distance) compared with the control and T2 groups on day 28 (Fig. 4G), while no significant differences were observed on day 14 (Fig. 4F).

Principal component analysis showed that the fecal microflora in the control, T1, and T2 piglets were grouped according to the dietary treatments and the age (Fig. 4C). The tree of the relative abundance of fecal microflora community also confirmed this result (Fig. 5H). Firmicutes and Bacteroidetes were the most 2 dominant phyla, among the phyla that were represented including Tenericutes, Proteobacteria, and Actinobacteria. Furthermore, Lactobacillus and Streptococcus were the 2 top species among the recognized microbiota (Fig. 5I).

The relative abundance of top 2 Lactobacillus species is listed in Fig. 5A and B. Compared with the control piglets, the T1 piglets had a higher relative abundance of Lactobacillus salivarius on day 14 and 28, but no statistically significant difference was observed. Interestingly, the supplementation with essential oil significantly increased the relative 

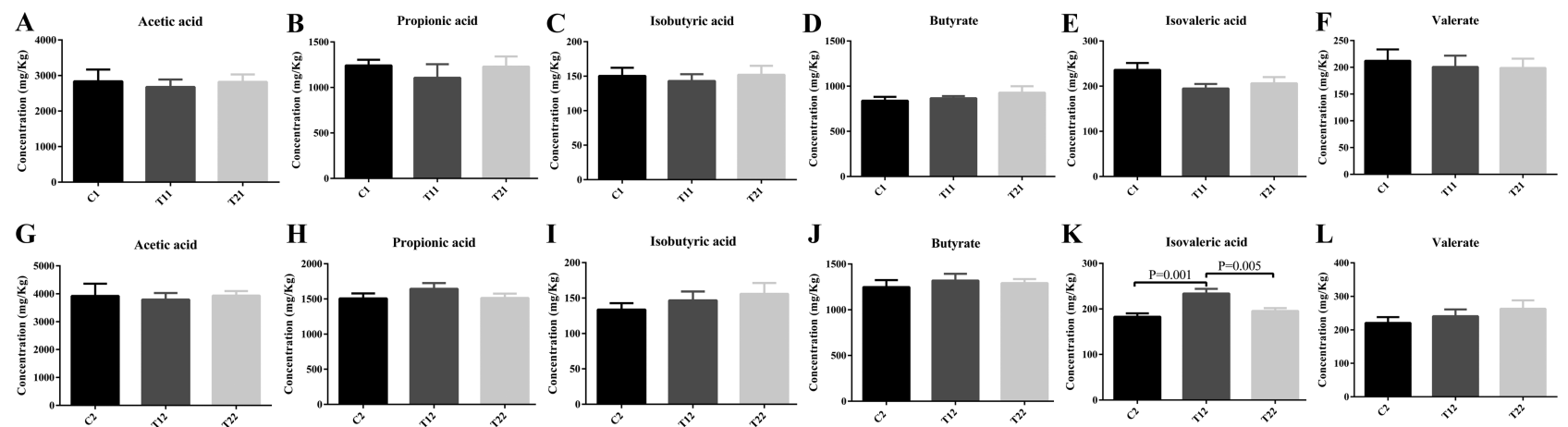

Figure 3. Effects of the combination of essential oils and organic acids on the fecal concentration of major volatile fatty acids in weaned piglets. $\mathrm{C} 1$ and $\mathrm{C} 2$ represent the control piglets on day 14 and 28, respectively; T11 and T12 represent the piglets supplemented with combination of essential oils and organic acids on day 14 and 28, respectively; T21 and T22 represent the antibiotics piglets on day 14 and 28 , respectively. Small superscript letter are different $(P<0.05)$. Values mean $n=10$ for the analysis of VFAs.

Table 3. Effects of the combination of essential oils and organic acids on the levels of serum immunoglobulins, C3, C4, and TNF- $\alpha$ in weaned piglets ${ }^{1}$

\begin{tabular}{|c|c|c|c|c|c|c|}
\hline \multirow[b]{2}{*}{ Parameter } & \multirow[b]{2}{*}{ Time (d) } & \multicolumn{3}{|c|}{ Treatment } & \multirow[b]{2}{*}{ SEM $^{2}$} & \multirow[b]{2}{*}{$P$ value } \\
\hline & & $\mathrm{C}$ & $\mathrm{T} 1$ & $\mathrm{~T} 2$ & & \\
\hline \multirow[t]{2}{*}{$\mathrm{IgA}, \mathrm{mg} / \mathrm{mL}$} & 14 & 4.94 & 6.25 & 6.45 & 0.25 & 0.11 \\
\hline & 28 & 5.84 & 6.83 & 6.47 & 0.26 & 0.59 \\
\hline \multirow[t]{2}{*}{$\mathrm{IgG}, \mathrm{mg} / \mathrm{mL}$} & 14 & 15.57 & 16.21 & 15.94 & 0.51 & 0.89 \\
\hline & 28 & 16.00 & 16.46 & 16.19 & 0.74 & 0.97 \\
\hline \multirow[t]{2}{*}{$\mathrm{IgM}, \mathrm{mg} / \mathrm{mL}$} & 14 & 7.59 & 8.57 & 7.58 & 0.37 & 0.49 \\
\hline & 28 & 6.39 & 6.72 & 6.92 & 0.45 & 0.90 \\
\hline \multirow[t]{2}{*}{$\mathrm{C} 3, \mu \mathrm{g} / \mathrm{mL}$} & 14 & 143.50 & 149.41 & 182.20 & 10.08 & 0.25 \\
\hline & 28 & 154.66 & 184.22 & 199.28 & 8.79 & 0.10 \\
\hline \multirow[t]{2}{*}{$\mathrm{C} 4, \mu \mathrm{g} / \mathrm{mL}$} & 14 & 166.00 & 197.51 & 213.15 & 12.98 & 0.34 \\
\hline & 28 & $179.27^{\mathrm{b}}$ & $228.40^{\mathrm{a}}$ & $224.22^{\mathrm{a}}$ & 9.35 & 0.05 \\
\hline \multirow[t]{2}{*}{ TNF- $\alpha, n g / L$} & 14 & 218.39 & 232.42 & 221.33 & 9.53 & 0.84 \\
\hline & 28 & 282.34 & 315.73 & 332.81 & 14.41 & 0.37 \\
\hline
\end{tabular}

${ }^{a, b}$ Means within the same raw with different superscripts differ significantly $(P<0.05)$.

${ }^{1} \mathrm{C}$, T1, and T2 represents the piglets supplemented with basal diet (control), piglets supplemented with the mixture of essential oils and organic acids and piglets supplemented with antibiotics, respectively.

${ }^{2}$ Pooled SEM; $n=10$ per treatment.

abundance of Lactobacillus mucosae compared to $\mathrm{C} 2$ and $\mathrm{T} 2$ piglets on day $28(P<0.05)$.

\section{DISCUSSION}

Studies have confirmed that either dietary essential oils or organic acids could play beneficial effects on the growth and health in weanling pigs (Puvača et al., 2013; Diao et al., 2014). While, few studies about the effects of essential oils and organic acids on piglets' health were conducted by researchers. Our data showed that the synergistic antibacterial effects of essential oils and organic acids on pathogens were more effectively than that of essential oils or organic acids alone in in vitro study. Here, the present study was performed to assess whether a mixture of essential oils and organic acids could be developed as a potential alternative to antibiotics in the pig industry, which could reduce the troubles caused by weanling. Several studies found that the essential oils (especially carvacrol and thymol) played beneficial roles on the growth performance in pigs ( $\mathrm{Li}$ et al., 2012; Zeng et al., 2015). The current study revealed that dietary supplementation with the mixture of essential oils and organic acids significantly improved the final BW and ADG of weaned piglets, although it did not affect the ADFI, F:G, or the diarrheal incidence. While other studies reported that the effects of essential oils on feed intake of pigs were ambiguous, which feed intake change relative to control due to dietary supplementation of essential oils ranged from $-9 \%$ to $12 \%$ (Franz et al., 2010), and $-3 \%$ to $19 \%$ (Zeng et al., 2015). Hence, more studies are needed to performed to investigate the specific mechanisms of essential oils and organic oils applied into feeding piglets. 
A

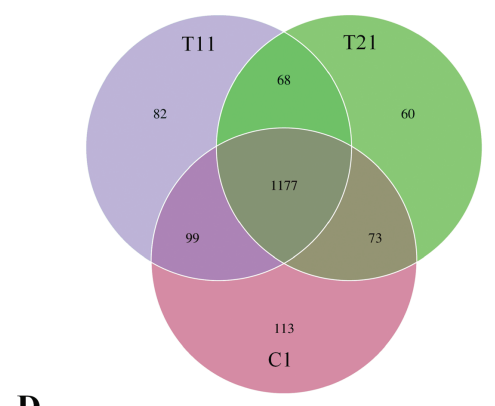

D

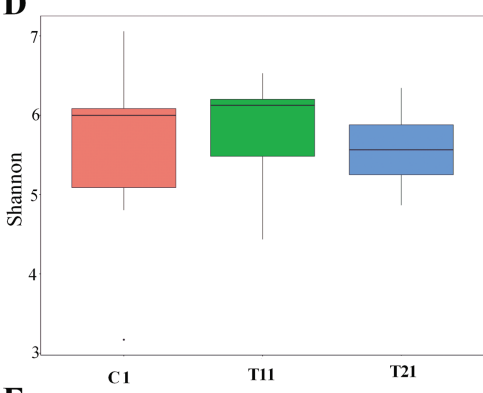

E

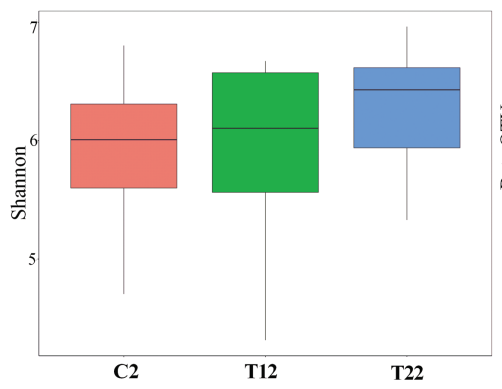

B

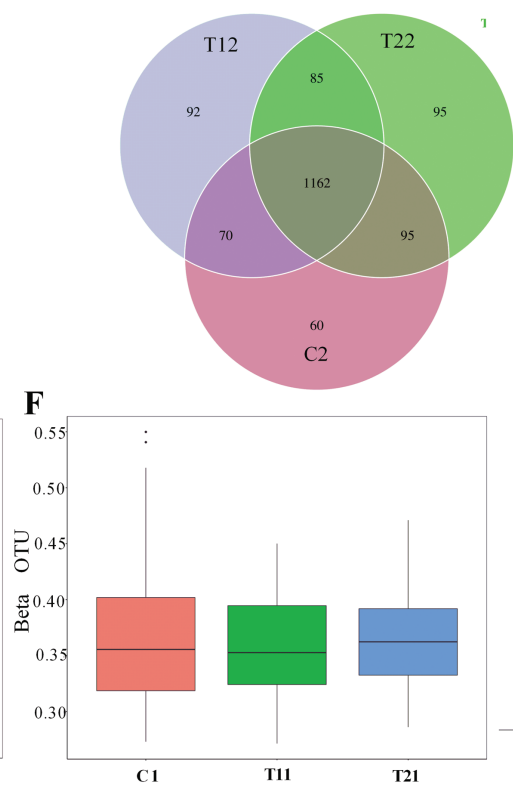

G

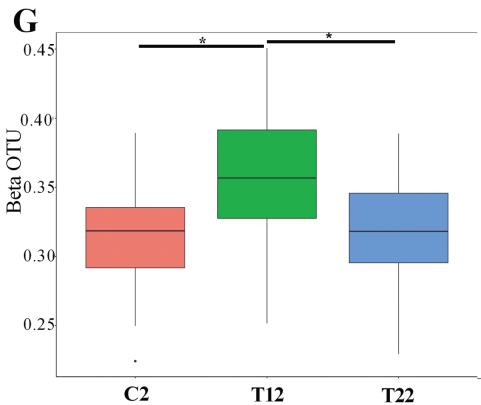

C

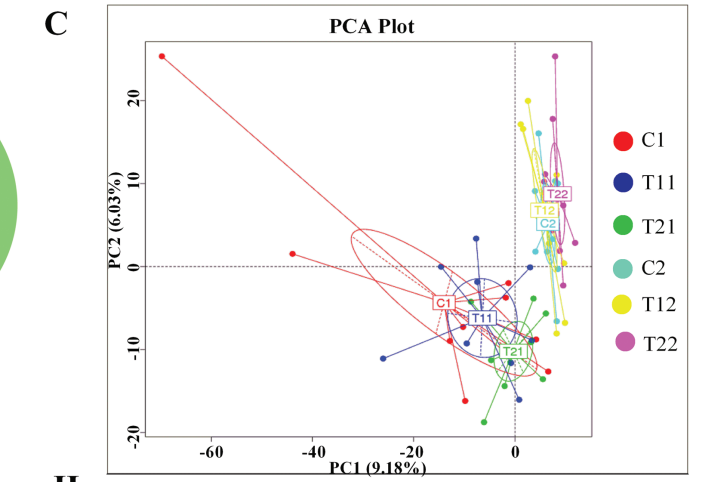

H

Relative Abundance in Phylum Level

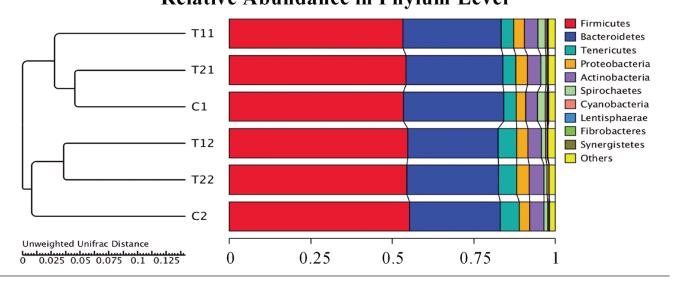

I

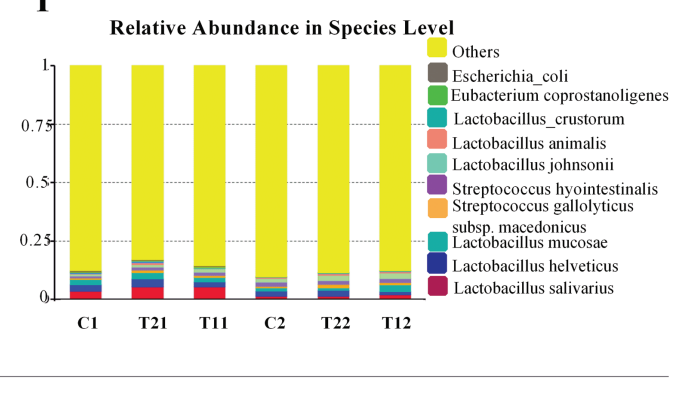

Figure 4. Summary of the fecal microbial communities in weaned piglets. A and B represent the Venn diagram summarizing the numbers of common and unique OTUs in the fecal microflora community of weaning piglets on day 14 and 28, respectively; C represents principle component analysis plot about the fecal microflora in weaning piglets; D and E represent the alpha diversity about the fecal microbial community of weaning piglets on day 14 and 28, respectively; F and $\mathrm{G}$ represent the beta diversity of the fecal microbial community in weaning piglets based on unweighted UniFrac distance on day 14 and 28, respectively; $\mathrm{H}$ represents the relative abundances of predominant bacteria at the phylum level in each group (unweighted UniFrac distance); I represents the top 10 relative abundance of fecal microflora community in weaning piglets (level species). $\mathrm{C} 1$ and $\mathrm{C} 2$ represents the control piglets on day 14 and 28, respectively; T11 and T12 represents the piglets supplemented with combination of essential oils and organic acids on day 14 and 28, respectively; T21 and T22 represents the antibiotics piglets on day 14 and 28 , respectively. OTU, operational taxonomic unit. Each treatment with $n=10$.

The major components of the essential oils that showed the highest antibacterial activity were aldehydes or phenols, such as cinnamaldehyde, citral, carvacrol, eugenol, and thymol (Bassole et al., 2012). Previous studies have confirmed that essential oils can damage the cellular structure of the pathogen, and can damage the bacterial cell membrane by altering the lipopolysaccharides of the cell membrane (Vaara, 1992; Sikkema et al., 1994). Fei et al. (2011) confirmed that the membranes' integrity of $E$. coli and $S$. aureus were significantly disrupted by the compound of essential oils. In other hand, Omonijo et al. (2018) found that the essential oils change the structure and permeability of the bacterial cell, which increases the chance of bacterial exposure to organic acids. A few recent studies also confirmed the synergism of dietary essential oils and organic acids in pigs (Balasubramanian et al., 2016; Walia et al., 2017; Liu et al., 2017). In the current study, a mixture of cinnamaldehyde and organic acid induced membrane deformation of the bacterial cell and disorganized intracellular structures, which are in agreement with the previous studies.

It is known that the VFAs, major metabolites of microbial fermentation, can offer an important energy source for gut epithelial cells, inhibit harmful bacteria by decreasing the intestinal $\mathrm{pH}$ values, and indirectly modulate the intestinal microflora (Corrier et al., 1990; Mountzouris et al., 2007). Bühler et al. (2009) found that the dietary benzoic acid increased the concentration of butyric acid in the intestine and regulated the gastrointestinal microflora. The supplementation of benzoic 
A

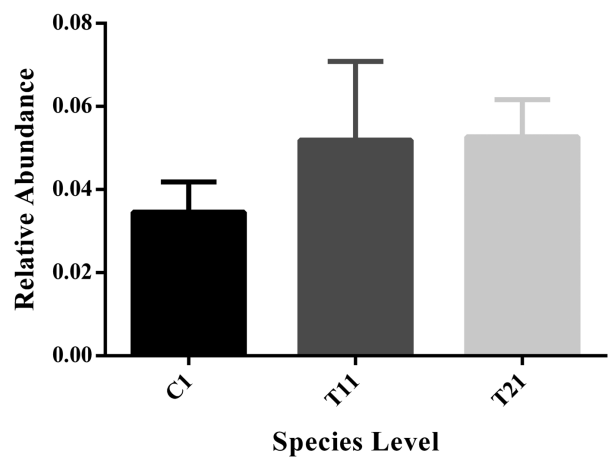

C

Lactobacillus Mucosae

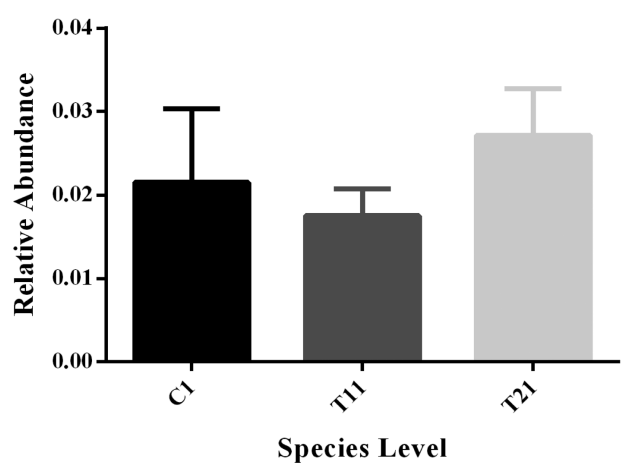

B

Lactobacillus Salivarius

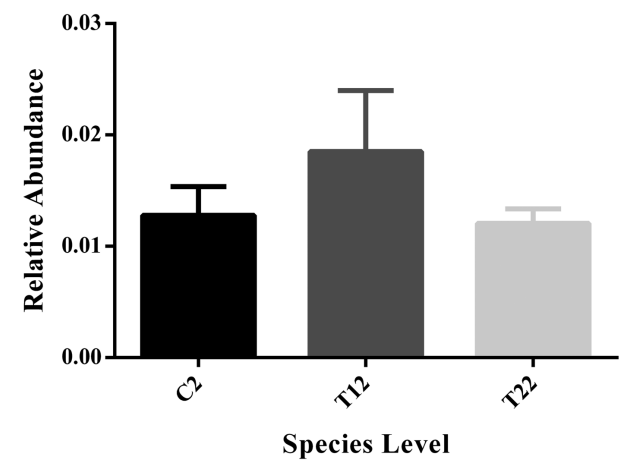

D

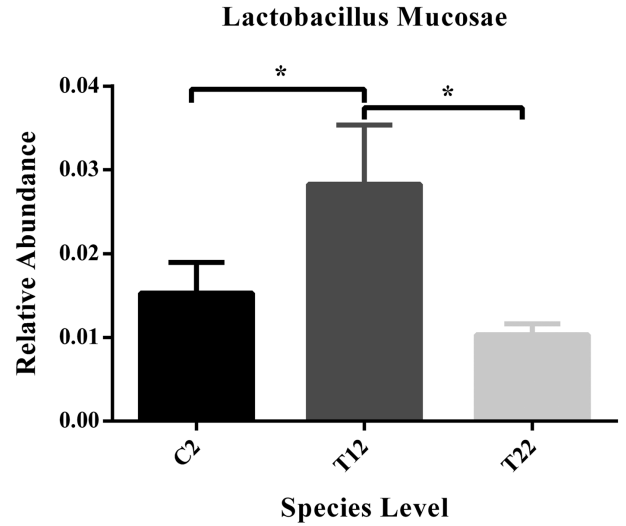

Figure 5. Changes of the relative abundance of Lactobacillus salivarius and Lactobacillus mucosae in the feces of piglets on the compound oil diets. $\mathrm{C} 1$ and $\mathrm{C} 2$ represent the control piglets on day 14 and 28, respectively; T11 and T12 represent the piglets supplemented with combination of essential oils and organic acids on day 14 and 28, respectively; T21 and T22 represent the antibiotics piglets on day 14 and 28 , respectively. Each treatment with $n=10$.

acid increased the concentration of total VFA and decreased the $\mathrm{pH}$ values of the cecum in pigs (Diao et al., 2014). A recently published study indicated that using mixed organic acids as feed additives led to a higher fecal concentration of total VAFs in piglets (Long et al., 2018). Li et al. (2018) also found that the dietary supplementation with organic oils improved the intestinal health by increasing the cecal levels of VFAs in the piglets. Our data showed that the concentration of isovaleric acid was significantly increased by the mixture of essential oils and organic acids in the feces of weaned piglets.

Immunoglobulins are required to regulate the immune function, and diminish weaning stress in weaned piglets (Lee et al., 2016). An increase in immunoglobulin levels might be beneficial in the postweaned pigs (Yoon et al., 2012). Previous studies have confirmed that the dietary supplementation with essential oils increased the levels of $\operatorname{IgA}$ and IgG (Zeng et al., 2015), C3 and C4 (Li et al., 2012), and lymphocytes in the lamina propria in pigs (Manzanilla et al., 2006). In this sense, essential oils can enhance the health by modulating the immune responses (Omonijo et al., 2018). Similarly, organic acids played a positive role in improving the immune system in pigs, particularly by enhancing the serum concentration of IgG (Cho et al., 2006; Ahmed et al., 2014). The current study showed that the dietary combination of essential oils and organic acids significantly enhanced the production of serum $\mathrm{C} 4$, increased serum $\mathrm{C} 3$ of weaned piglets at the end of experiment. On the contrary, a few studies on the effect of essential oil on immune system have been reported. Wei et al., (2017) reported an essential oil-mediated decrease in the expression of TNF- $\alpha$ mRNA (Wei et al., 2017), and a decrease in serum IgG (Yan et al., 2010). Ariza-Nieto et al. (2011) reported that essential oil supplementation did not improve the immune responses in pigs. Hence, it is difficult to conclude whether essential oils and organic acids could play a beneficial role on the gut immune system in weaned piglets, and therefore, additional studies are required to uncover the role of essential oils and organic acids in immune development.

Earlier studies have shown that Firmicutes, Bacteroidetes, Proteobacteria, and Actinobacteria were the most dominant phyla in pigs (Kim et al., 
2015; Zhao et al., 2015). The principle component analysis revealed that age and diet induced dynamic shifts in the structure of the microbial community. In this study, Firmicutes, Bacteroidetes, Tenericutes, Proteobacteria, and Actinobacteria were the most predominant phyla regardless of age, which was similar with the previous studies. Furthermore, essential oils can modulate the intestinal microflora ecosystem by preventing the proliferation of pathogenic bacteria and improve the overall status of health and immunity, resulting in an augmented productive performance (Pathak et al., 2016). Our data revealed that the dietary essential oils and organic acids increased the relative abundance of Lactobacilli in feces, which would produce more useful metabolites. Similarly, Xu et al. (2018) reported that the organic acid plus basal diet positively modulated the microbial populations by increasing the fecal Lactobacillus compared with antibiotic diet in weaned piglets, which may be relative to the improvement of proliferation of epithelial cells and intestinal morphology. Castillo et al. (2006) also found that plant extract mixture diet (carvacrol, cinnamaldehyde, and capsicum oleoresin) increased the ratio of Lactobacilli to Enterobacteria in the cecum of early-weaned pigs.

\section{CONCLUSION}

Dietary supplementation with a mixture of essential oils and organic acids damage the cellular structure of pathogenic bacteria, and significantly improve the growth performance, modulate the serum immune parameters, enhance the concentration of fecal isovaleric acid and alter the fecal microbial community in weaned piglets.

Conflict of interest statement. None declared

\section{ACKNOWLEDGMENTS}

The present research was supported by the Key Research Project of Zhejiang Province (No. 2017C02005) and the National Natural Science Foundation of China (No. 31471636, 31501985). We also acknowledge Vegamax Biotechnology Co. Ltd. (Anji, Zhejiang, China) for providing the oxytetracycline, the product of essential oil and organic acids.

\section{LITERATURE CITED}

Ahmed, S. T., J. A. Hwang, J. Hoon, H. S. Mun, and C. J. Yang. 2014. Comparison of single and blend acidifiers as alternative to antibiotics on growth performance, fecal microflora, and humoral immunity in weaned piglets. Asian-Australas. J. Anim. Sci. 27:93-100. doi:10.5713/ ajas.2013.13411
Amorati, R., M. C. Foti, and L. Valgimigli. 2013. Antioxidant activity of essential oils. J. Agric. Food Chem. 61:1083510847. doi:10.1021/jf403496k

Ariza-Nieto, C., M. Bandrick, S. K. Baidoo, L. Anil, T. W. Molitor, and M. R. Hathaway. 2011. Effect of dietary supplementation of oregano essential oils to sows on colostrum and milk composition, growth pattern and immune status of suckling pigs. J. Anim. Sci. 89:1079-1089. doi:10.2527/jas.2010-3514

Balasubramanian, B., J. W. Park, and I. H. Kim. 2016. Evaluation of the effectiveness of supplementing micro-encapsulated organic acids and essential oils in diets for sows and suckling piglets. Ital. J. Anim. Sci. 15:626-633. doi:10 $.1080 / 1828051 X .2016 .1222243$

Bassolé, I. H., and H. R. Juliani. 2012. Essential oils in combination and their antimicrobial properties. Molecules 17:3989-4006. doi:10.3390/molecules17043989

Boudry, G., V. Péron, I. Le Huërou-Luron, J. P. Lallès, and B. Sève. 2004. Weaning induces both transient and long-lasting modifications of absorptive, secretory, and barrier properties of piglet intestine. J. Nutr. 134:22562262. doi: $10.1093 / \mathrm{jn} / 134.9 .2256$

Boudry, G., and C. Perrier. 2008. Thyme and cinnamon extracts induce anion secretion in piglet small intestine via cholinergic pathways. J. Physiol. Pharmacol. 59:543-552.

Bühler, K., B. Bucher, C. Wenk, and J. Broz. 2009. Influence of benzoic acid in high fibre diets on nutrient digestibility and vfa production in growing/finishing pigs. Arch. Anim. Nutr. 63(2):127-136. doi:10.1080/17450390902723927

Cairo, P. L. G., F. D. Gois, M. Sbardella, H. Silveira, R. M. de Oliveira, I. B. Allaman, V. S. Cantarelli, and L. B. Costa. 2018. Effects of dietary supplementation of red pepper (Schinus terebinthifolius raddi) essential oil on performance, small intestinal morphology and microbial counts of weanling pigs. J. Sci. Food Agric. 98:541-548. doi:10.1002/jsfa.8494

Castillo, M., S. M. Martín-Orúe, M. Roca, E. G. Manzanilla, I. Badiola, J. F. Perez, and J. Gasa. 2006. The response of gastrointestinal microbiota to avilamycin, butyrate, and plant extracts in early-weaned pigs. J. Anim. Sci. 84:27252734. doi:10.2527/jas.2004-556

Cho, J. H., Y. J. Chen, B. J. Min, H. J. Kim, O. S. Kwon, K. S. Shon, I. H. Kim, S. J. Kim, and A. Asamer. 2006. Effects of essential oils supplementation on growth performance, IgG concentration and fecal noxious gas concentration of weaned pigs. Asian-Australas. J. Anim. Sci . 19:80-85. doi:10.5713/ajas.2006.80

Corrier, D. E., A. Hinton, Jr, R. L. Ziprin, and J. R. DeLoach. 1990. Effect of dietary lactose on salmonella colonization of market-age broiler chickens. Avian Dis. 34:668-676.

Cui, B., D. Su, W. Li, X. She, M. Zhang, R. Wang, and Q. Zhai. 2018. Effects of chronic noise exposure on the microbiome-gut-brain axis in senescence-accelerated prone mice: implications for Alzheimer's disease. J. Neuroinflammation. 15:190. doi:10.1186/s12974-018-1223-4

Diao, H., P. Zheng, B. Yu, J. He, X. B. Mao, J. Yu, and D. W. Chen. 2014. Effects of dietary supplementation with benzoic acid on intestinal morphological structure and microflora in weaned piglets. Livest. Sci. 167:249-256. doi:10.1016/j.livsci.2014.05.029

Fei, L., H. Liang, Q. Yuan, and C. Li. 2011. In vitro antimicrobial effects and mechanism of action of selected plant essential oil combinations against four food-related microorganisms. Food Res. Int. 44:3057-3064. doi:10.1016/j. foodres.2011.07.030 
Franz, C., K. Baser, and W. Windisch. 2010. Essential oils and aromatic plants in animal feeding - a European perspective. A review. Flavour. Frag. J. 25:327-340. doi:10.1002/ffj.1967

Hippenstiel, F., A. Abdel-Wareth, S. Kehraus, and K. Südekum. 2011. Effects of selected herbs and essential oils, and their active components on feed intake and performance of broilers-a review. Arch.Geflügelk. 75:S. 226-234.

Kim, J., S. G. Nguyen, R. B. Guevarra, I. Lee, and T. Unno. 2015. Analysis of swine fecal microbiota at various growth stages. Arch. Microbiol. 197:753-759. doi:10.1007/ s00203-015-1108-1

Lee, I. K., Y. C. Kye, G. Kim, H. W. Kim, M. J. Gu, J. Umboh, K. Maaruf, S. W. Kim, and C. H. Yun. 2016. Stress, nutrition, and intestinal immune responses in pigs - A review. Asian-Australas. J. Anim. Sci. 29:1075-1082. doi:10.5713/ ajas. 16.0118

Li, P. F., X. S. Piao, Y. J. Ru, X. Han, L. F. Xue, and H. Y. Zhang. 2012. Effects of adding essential oil to the diet of weaned pigs on performance nutrient utilization, immune response and intestinal health. AsianAustralas. J. Anim. Sci. 25:1617-1626. doi:10.5713/ ajas.2012.12292

Li, S., J. Zheng, K. Deng, L. Chen, X. L. Zhao, X. Jiang, Z. Fang, L. Che, S. Xu, B. Feng, et al. 2018. Supplementation with organic acids showing different effects on growth performance, gut morphology, and microbiota of weaned pigs fed with highly or less digestible diets. J. Anim. Sci. 96:3302-3318. doi:10.1093/jas/sky197

Liu, Y., L. He, A. Mustapha, H. Li, Z. Q. Hu, and M. Lin. 2009. Antibacterial activities of zinc oxide nanoparticles against Escherichia coli O157:H7. J. Appl. Microbiol. 107:1193-1201. doi:10.1111/j.1365-2672.2009.04303.X

Liu, Y., X. Yang, H. Xin, S. Chen, C. Yang, Y. Duan, and X. Yang. 2017. Effects of a protected inclusion of organic acids and essential oils as antibiotic growth promoter alternative on growth performance, intestinal morphology and gut microflora in broilers. Anim. Sci. J. 88:1414-1424. doi:10.1111/asj.12782

Long, S. F., Y. T. Xu, L. Pan, Q. Q. Wang, C. L. Wang, J. Y. Wu, Y. M. Han, C. H. Yun, and X. S. Piao. 2018. Mixed organic acids as antibiotic substitutes improve performance, serum immunity, intestinal morphology and microbiota for weaned piglets. Anim. Feed. Sci. Technol. 235:23-32. doi:10.1016/j.anifeedsci.2017.08.018

Manzanilla, E. G., M. Nofrarías, M. Anguita, M. Castillo, J. F. Perez, S. M. Martín-Orúe, C. Kamel, and J. Gasa. 2006. Effects of butyrate, avilamycin, and a plant extract combination on the intestinal equilibrium of early-weaned pigs. J. Anim. Sci. 84:2743-2751. doi:10.2527/jas.2005-509

Mountzouris, K. C., P. Tsirtsikos, E. Kalamara, S. Nitsch, G. Schatzmayr, and K. Fegeros. 2007. Evaluation of the efficacy of a probiotic containing lactobacillus, bifidobacterium, enterococcus, and pediococcus strains in promoting broiler performance and modulating cecal microflora composition and metabolic activities. Poult. Sci. 86:309317. doi:10.1093/ps/86.2.309

Mulyaningsih, S., M. Youns, M. Z. El-Readi, M. L. Ashour, E. Nibret, F. Sporer, F. Herrmann, J. Reichling, and M. Wink. 2010. Biological activity of the essential oil of kadsura longipedunculata (schisandraceae) and its major components. J. Pharm. Pharmacol. 62:1037-1044. doi:10.1111/j.2042-7158.2010.01119.x

Omonijo, F. A., L. Ni, J. Gong, Q. Wang, L. Lahaye, and
C. Yang. 2018. Essential oils as alternatives to antibiotics in swine production. Anim. Nutr. 4:126-136. doi:10.1016/j. aninu.2017.09.001

Pathak, M., G. P. Mandal, A. K. Patra, I. Samanta, S. Pradhan and S. Haldar. 2016. Effects of dietary supplementation of cinnamaldehyde and formic acid on growth performance, intestinal microbiota and immune response in broiler chickens. Anim. Prod. Sci. 57(5):821-827. doi:10.1071/ AN15816

Puvača, N., V. Stanaćev, D. Glamočić, J. Lević, L. Perić, and D. MILIĆ. 2013. Beneficial effects of phytoadditives in broiler nutrition. World's. Poult. Sci. J. 69:27-34. doi:10.1017/S0043933913000032

Sikkema, J., J. A. de Bont, and B. Poolman. 1994. Interactions of cyclic hydrocarbons with biological membranes. J. Biol. Chem. 269:8022-8028.

Suiryanrayna, M., and J. V. Ramana. 2016. A review of the effects of dietary organic acids fed to swine. J. Anim. Sci. Biotechnol. 6:45. doi:10.1186/s40104-015-0042-z

Sun, P., D. Li, Z. Li, B. Dong, and F. Wang. 2008. Effects of glycinin on IgE-mediated increase of mast cell numbers and histamine release in the small intestine. J. Nutr. Biochem. 19:627-633. doi:10.1016/j. jnutbio.2007.08.007.

Thanh, N. T., T. C. Loh, H. L. Foo, M. Hair-Bejo, and B. K. Azhar. 2009. Effects of feeding metabolite combinations produced by lactobacillus plantarum on growth performance, faecal microbial population, small intestine villus height and faecal volatile fatty acids in broilers. Br. Poult. Sci. 50:298-306. doi:10.1080/00071660902873947.

Torrallardona, D., I. Badiola, and J. Broz. 2007. Effects of benzoic acid on performance and ecology of gastrointestinal microbiota in weanling piglets. Livest. Sci. 108:210-213. doi:10.1016/j.livsci.2007.01.062

Vaara, M. 1992. Agents that increase the permeability of the outer membrane. Microbiol. Rev. 56:395-411.

Valenzuela-Grijalva, N. V., A. Pinelli-Saavedra, A. MuhliaAlmazan, D. Domínguez-Díaz, and H. González-Ríos. 2017. Dietary inclusion effects of phytochemicals as growth promoters in animal production. J. Anim. Sci. Technol. 59:8. doi:10.1186/s40781-017-0133-9.

Walia, K., H. Argüello, H. Lynch, F. C. Leonard, J. Grant, D. Yearsley, S. Kelly, G. Duffy, G. E. Gardiner, and P. G. Lawlor. 2017. Effect of strategic administration of an encapsulated blend of formic acid, citric acid, and essential oils on salmonella carriage, seroprevalence, and growth of finishing pigs. Prev. Vet. Med. 137(Pt A):28-35. doi:10.1016/j.prevetmed.2016.12.007.

Wei, H. K., H. X. Xue, Z. X. Zhou, and J. Peng. 2017. A carvacrol-thymol blend decreased intestinal oxidative stress and influenced selected microbes without changing the messenger RNA levels of tight junction proteins in jejunal mucosa of weaning piglets. Animal. 11:193-201. doi:10.1017/S1751731116001397.

Xu, Y. T., L. Liu, S. F. Long, L. Pan, and X. S. Piao. 2018. Effect of organic acids and essential oils on performance, intestinal health and digestive enzyme activities of weaned pigs. Anim. Feed Sci. Technol. 235:110-119. doi:10.1016/j. anifeedsci.2017.10.012

Yan, L., J. Wang, H. Kim, Q. Meng, X. Ao, S. M. Hong, and I. H. Kim. 2010. Influence of essential oil supplementation and diets with different nutrient densities on growth performance, nutrient digestibility, blood characteristics, 
meat quality and fecal noxious gas content in grower-finisher pigs. Livest. Sci. 128:115-122. doi:10.1016/j. livsci.2009.11.008

Yang, C., M. A. Chowdhury, Y. Huo, and J. Gong. 2015. Phytogenic compounds as alternatives to in-feed antibiotics: potentials and challenges in application. Pathogens. 4:137-156. doi:10.3390/pathogens4010137

Yoon, J. H., S. L. Ingale, J. S. Kim, K. H. Kim, S. H. Lee, Y. K. Park, I. K. Kwon, and B. J. Chae. 2012. Effects of dietary supplementation of antimicrobial peptide-A3 on growth performance, nutrient digestibility, intestinal and fecal microflora and intestinal morphology in weanling pigs. Anim. Feed. Sci. Technol. 177:98-107. doi:10.1016/j. nifeedsci.2012.06.009

Zeng, Z., X. Xu, Q. Zhang, P. Li, P. Zhao, Q. Li, J. Liu, and X. Piao. 2015. Effects of essential oil supplementation of a low-energy diet on performance, intestinal morphology and microflora, immune properties and antioxidant activities in weaned pigs. Anim. Sci. J. 86:279-285. doi:10.1111/ asj.12277.

Zhu, B. K., Y. M. Fang, D. Zhu, P. Christie, X. Ke, and Y. G. Zhu. 2018. Exposure to nanoplastics disturbs the gut microbiome in the soil oligochaete enchytraeus crypticus. Environ. Pollut. 239:408-415. doi:10.1016/j. envpol.2018.04.017 